\title{
COMPOSITION OPERATORS ON BANACH SPACES OF ANALYTIC FUNCTIONS
}

\author{
Mieczysław Mastyło and Paweł Mleczko \\ Adam Mickiewicz University Poznań, Faculty of Mathematics and Computer Science \\ Umultowska 87, 61-614 Poznań, Poland; mastylo@amu.edu.pl \\ Adam Mickiewicz University Poznań, Faculty of Mathematics and Computer Science \\ Umultowska 87, 61-614 Poznań, Poland; pml@amu.edu.pl
}

\begin{abstract}
In the paper composition operators acting on quasi-Banach spaces of analytic functions on the unit disc of the complex plane are studied. In particular characterizations in terms of a function $\varphi$ of order bounded as well as summing operators $C_{\varphi}$ are presented, if $C_{\varphi}$ is an operator from an abstract Hardy space. Applications are shown for the special case of Hardy-Orlicz, Hardy-Lorentz, and growth spaces.
\end{abstract}

\section{Introduction}

Let $\mathbf{D}$ be the unit disc of the complex plane $\mathbf{C}$ and denote by $H(\mathbf{D})$ the space of holomorphic functions on D. For a function $\varphi \in H(\mathbf{D})$ satisfying $\varphi(\mathbf{D}) \subset \mathbf{D}$ the composition operator $C_{\varphi}: H(\mathbf{D}) \rightarrow H(\mathbf{D})$ is defined with the formula

$$
C_{\varphi} f(z)=f \circ \varphi(z), \quad f \in H(\mathbf{D}), z \in \mathbf{D} .
$$

The composition operator $C_{\varphi}: \mathcal{X}(\mathbf{D}) \rightarrow H(\mathbf{D})$ restricted to any subspace $\mathcal{X}(\mathbf{D})$ of $H(\mathbf{D})$ consisting of functions having radial limits, induces an operator $\widetilde{C}_{\varphi}$ mapping $\mathcal{X}(\mathbf{D})$ into the space of Borel measurable functions given by $\widetilde{C}_{\varphi} f:=\widetilde{C_{\varphi} f}$ for all $f \in \mathcal{H}(\mathbf{D})$, where $\widetilde{g}$ denotes the radial limit function of $g \in \mathcal{H}(\mathbf{D})$.

This paper is devoted to the study of composition operators on abstract variants of Hardy spaces. The most research has been done on the composition operators on the Hilbert-Hardy space $H^{2}$ and its direct generalizations-Hardy classes $H^{p}$. Note however that in the last years the study of composition operators in the more general variants of Banach spaces of holomorphic functions on $\mathbf{D}$ have attracted more attention, just to mention the recent memoir [12] and articles [6, 13, 16, 17].

The study of composition operators on spaces of analytic functions reveals remarkable and enlightening connections between operator theoretic properties of $C_{\varphi}$ and geometric properties of the symbol $\varphi$. We refer the reader to Shapiro's article [20] and the comments given there. In this note we present a further evidence of this principle and show how to study order boundedness and summability of composition operators acting on general Hardy spaces. Our research at its bases dates back to the fundamental paper of Shapiro and Taylor [21], where the study of summing composition operators on spaces $H^{p}$ were initiated. Let us remark that this research was then expanded in several papers including articles of Hunziker and Jarchow [5], Jarchow [7, 8, 9], Jarchow and Riedl [10], Domenig [3], and recently by Lefèvre and

https://doi.org/10.5186/aasfm.2019.4436

2010 Mathematics Subject Classification: Primary 47B33, 47B38; Secondary 47B10.

Key words: Hardy space, interpolation space, composition operators, absolutely summing operators, growth spaces, order bounded operators.

The work was supported by The Foundation for Polish Science (FNP). 
Rodríguez-Piazza in [14]. Note that in the mentioned papers the study of absolutely summing composition operators on $H^{p}$ was combined with the study of factorization of operators and order boundedness.

Our research goes far beyond the classical spaces. In this paper we use functional analysis techniques and interpolation theory to study absolutely summing composition and order boundedness operators on abstract variants of Hardy spaces. Our approach is similar in its philosophy to the one presented in the recent article [17], where the Carleson embeddings from abstract Hardy spaces into Banach lattices were analyzed.

In particular we will study order bounded composition operators on the so-called growth spaces. Let $\phi:[0,1] \rightarrow[0, \infty)$ be a continuous decreasing function with $\phi(1)=$ 0 and $\phi(r)>0$ for $r \in[0,1)$. We define the Banach space $X_{\phi}$ of all analytic functions $f: \mathbf{D} \rightarrow \mathbf{C}$ equipped with the norm

$$
\|f\|_{X_{\phi}}=\sup _{z \in \mathbf{D}} \phi(|z|)|f(z)| .
$$

Let us note the special case when for $0<p<\infty, \phi(r)=(1-r)^{1 / p}$, for all $r \in[0,1]$, was studied by Jarchow and Riedl in [10]. In what follows when $\phi(r)=(1-r)^{1 / p}$, we write $X_{1 / p}$ instead of $X_{\phi}$.

The main results of the paper are contained in Sections 3 and 4 . For the convenience we present below the flavor of those outcomes. In Section 3 we study order bounded operators $\widetilde{C}_{\varphi}$ from growth spaces $X_{\phi}$ into quasi-Banach lattices on $[0,2 \pi)$. The main result states that if $Y$ has a non-trivial convexity and $\psi$ be a quasi-concave function satisfying some mild condition, then the operator $\widetilde{C}_{\varphi}$ is order bounded from $X_{\phi}$ into $Y$ with $\phi(r)=1 / \psi\left(\frac{1}{1-r}\right)$ for all $r \in[0,1)$ if and only if one of the following equivalent condition is satisfied: $\psi\left(\frac{1}{1-|\widetilde{\varphi}|}\right)$ belongs to $Y$ or $\widetilde{C}_{\varphi}$ defines a bounded operator from $X_{\phi}$ into $Y$.

The results of Section 4 are inspired by Shapiro and Taylor paper [21], where it was shown that the composition operator $C_{\varphi}$ is $p$-absolutely summing provided that $(1-|\widetilde{\varphi}|)^{-1}$ is integrable on $[0,2 \pi)$. We investigate more general summing property. Combining general variants of Hausdorff-Young inequalities with interpolation, we study abstract summing and order bounded composition operators $C_{\varphi}$ on general Hardy spaces. We present applications to Hardy-Orlicz and Hardy-Lorentz spaces. In particular as a by-product of general results we show that if $p \in[2, \infty)$ and $1 / p+1 / p^{\prime}=1$, then $\widetilde{C}_{\varphi}$ defines an order bounded operator from the Hardy-Lorentz space $H_{p, p^{\prime}}$ into $L^{p}$ if and only if one of the following equivalent condition is satisfied: $(1-|\widetilde{\varphi}|)^{-1}$ is integrable on $[0,2 \pi)$ or $\widetilde{C}_{\varphi}$ defines a $p$-summing operator from $H_{p, p^{\prime}}$ into $L^{p}$.

\section{Preliminaries}

Throughout the paper we will use the standard notations from the Banach space theory and the theory of Hardy spaces. Below we collect the basic notions and definitions.

Symmetric quasi-Banach spaces. If $X$ is a quasi-Banach space we denote by $B_{X}$ the closed unit ball of $X$. For a given complete $\sigma$-finite measure space $(\Omega, \mu):=$ $(\Omega, \Sigma, \mu)$ the symbol $L^{0}(\mu):=L^{0}(\Omega, \Sigma, \mu)$ stands for the space of (equivalence classes of $\mu$-a.e. equal) complex-valued measurable functions on $\Omega$ with the topology of 
convergence in measure on $\mu$-finite sets. As usual the order $|f| \leq|g|$ means that $|f(t)| \leq|g(t)|$ for $\mu$-almost all $t \in \Omega$.

If a quasi-normed space $X \subset L^{0}(\mu)$ is such that there exists $u \in X$ with $u>0 \mu$ a.e. on $\Omega$ and $X$ is solid (meaning that $f \in X$ with $\|f\|_{X} \leq\|g\|_{X}$ whenever $|f| \leq|g|$ with $f \in L^{0}(\mu)$ and $g \in X$ ), then $X$ is said to be a quasi-normed lattice (on $\Omega$ or on $(\Omega, \mu)$ ). A quasi-Banach lattice $X$ is said to be maximal (or to have the Fatou property), if for any sequence $\left\{f_{n}\right\}$ of non-negative elements from $X$ such that $f_{n} \uparrow f$ for $f \in L^{0}(\Omega)$ and $\sup \left\{\left\|f_{n}\right\|_{X}: n \in \mathbf{N}\right\}<\infty$, one has $f \in X$ and $\left\|f_{n}\right\|_{X} \rightarrow\|f\|_{X}$. A Banach lattice $X$ is said to be order continuous if for every $0 \leq f_{n} \downarrow 0$ a.e. it follows that $\left\|f_{n}\right\|_{X} \rightarrow 0$. If $X$ is an order-continuous Banach lattice on $(\Omega, \mu)$, then $X^{*}$ can be identified with the Köthe dual space $\left(X^{\prime},\|\cdot\|_{X^{\prime}}\right)$ of all $f \in L^{0}(\mu)$ such that

$$
\|f\|_{X^{\prime}}:=\sup \left\{\int_{\Omega}|f g| \mathrm{d} \mu:\|g\|_{X} \leq 1\right\}<\infty .
$$

We note that $X^{\prime}$ is a maximal Banach lattice on $(\Omega, \mu)$. It is well known that $X$ is maximal if and only if the Köthe bidual $X^{\prime \prime}=X$ with equality of norms. For details we refer the reader to [15].

An important class of quasi-Banach lattices on a measure space $(\Omega, \Sigma, \mu)$ are symmetric quasi-Banach spaces. Given $f \in L^{0}(\mu)$, its distribution function is defined as a function of non-negative $\lambda$ by $\mu_{f}(\lambda)=\mu\{t \in \Omega:|f(t)|>\lambda\}$. Then a quasiBanach lattice $X$ on $(\Omega, \mu)$ is said to be a symmetric quasi-Banach space if $g \in X$ and $\|f\|_{X}=\|g\|_{X}$ whenever $f \in X, g \in L^{0}$ and $\mu_{f}=\mu_{g}$. Symmetric Banach spaces are also called rearrangement invariant spaces (see [15]). In the sequel, if $X$ is a symmetric Banach space, we call it simply a symmetric space. Recall also that in this case $X \subset L^{1}(\mu)+L^{\infty}(\mu)$.

In what follows the term complex quasi-normed (resp., quasi-Banach) lattice refers to the complexification $X(\mathbf{C})$ of a real quasi-normed (resp., quasi-Banach) lattice $X$ (namely the space of all complex valued measurable functions $f$ on $\Omega$ such that the element $|f|$ defined by $|f|(t):=|f(t)|$ for $t \in \Omega$ belongs to $X$ and $\left.\|f\|_{X(\mathbf{C})}:=\||f|\|_{X}\right)$. Throughout the paper for the simplicity of presentation we will often avoid using the term "complex".

Hardy spaces on the disc. For any $f \in H(\mathbf{D})$ and $r \in[0,1)$, denote by $f_{r}$ the function $f_{r}: \overline{\mathbf{D}} \rightarrow \mathbf{C}$ given with the formula $f_{r}(z)=f(r z), z \in \overline{\mathbf{D}}$. Let $X$ be a symmetric space on $(\mathbf{T}, m)$, where $m$ is the normalized Lebesgue measure on $\mathbf{T}:=[0,2 \pi)$, that is $\mathrm{d} m\left(e^{i t}\right)=\frac{1}{2 \pi} \mathrm{d} t$. We define the abstract Hardy space $H X$ to be the Banach space of all $f \in H(\mathbf{D})$ such that $\sup \left\{\left\|f_{r}\right\|_{X}: r \in[0,1)\right\}<\infty$, equipped with the norm

$$
\|f\|_{H X}=\sup \left\{\left\|f_{r}\right\|_{X}: r \in[0,1)\right\} .
$$

We note that in the case when $X=L^{p}(m)$ and $p \in[1, \infty]$, then $H L^{p}$ is the classical Hardy space $H^{p}$ (we refer to the Duren's monograph [4]). Record that spaces $H X$ were studied for example in [12] for the case when $X$ is an Orlicz space and in more general case of $X$ being a symmetric space in [16].

As in the classical case of $H^{p}$ spaces, the fact that one can study properties of $H X$ functions via the study of the corresponding boundary $X$ functions, is very fruitful. The space of all $f \in H(\mathbf{D})$ such that the radial limit function

$$
\widetilde{f}(\xi):=\lim _{r \rightarrow 1^{-}} f_{r}(\xi)
$$


exists for $m$-almost every $\xi \in \mathbf{T}$, is denoted by $\widetilde{\mathcal{H}}(\mathbf{D})$. Since $H X \hookrightarrow H^{1}$ then for every function $f(z)=\sum_{n=0}^{\infty} a_{n} z^{n}$ in $H X$, the radial limit $\widetilde{f}$ exists a.e. on $\mathbf{T}$ (see [4, Theorem 5, p. 17]). In the sequel we will use the following fact without any references (see [16, Proposition 2.2]). If $X$ is a symmetric space on $\mathbf{T}$, then $f \in H X$ implies $\widetilde{f} \in X^{\prime \prime}$, the Köthe bidual of $X$, and

$$
\|f\|_{H X}=\|\widetilde{f}\|_{X^{\prime \prime}}
$$

In particular, in the case when $X$ is a maximal symmetric space (that is, when $X=X^{\prime \prime}$ ), the liner map $f \mapsto \tilde{f}$ is a linear isometric isomorphism from $H X$ onto a closed subspace of $X$, which consists of those members of $X$ whose negative Fourier coefficients vanish.

\section{Order bounded composition operators}

Throughout the paper $\Phi$ denotes the set of all analytic self-maps $\varphi: \mathbf{D} \rightarrow \mathbf{D}$. If $\varphi \in \Phi$ with $\left|\widetilde{\varphi}\left(e^{i t}\right)\right|<1$ for almost all $t \in \mathbf{T}$, we write $\varphi \in \widetilde{\Phi}$. From the Littlewood subordination principle, it follows that $C_{\varphi}$ maps every Hardy space $H^{p}, p>0$, into itself and is continuous on $H^{p}$ (see [4, Theorem 1.7]).

Let $\mathcal{X}(\mathbf{D}) \subset \widetilde{\mathcal{H}}(\mathbf{D})$ be a Banach space of analytic functions. Then every composition operator $C_{\varphi}: \mathcal{X}(\mathbf{D}) \rightarrow \mathcal{X}(\mathbf{D})$ induces an operator $\widetilde{C}_{\varphi}: \mathcal{X}(\mathbf{D}) \rightarrow L^{0}(m)$ defined by $\widetilde{C}_{\varphi} f=\widetilde{C_{\varphi}(f)}$. In this section we study order boundedness of operator $\widetilde{C}_{\varphi}$ defined on abstract spaces of analytic functions.

We recall that an operator $T: X \rightarrow Y$ from a Banach space $X$ into a Banach lattice $Y$ is order bounded if it maps the unit ball $B_{X}$ of $X$ into an order bounded interval of $Y$, i.e., there exists $y \in Y$ such that $|T x| \leq y$ for all $x \in B_{X}$.

Recall that the fundamental function $\varphi_{X}$ of a symmetric space $X(\mu)$ is given by $\varphi_{X}(t):=\left\|\chi_{A}\right\|_{X}$, where $A$ is any measurable set with $\mu(A)=t$. Notice that if $X$ is a symmetric space on nonatomic measure space $(\Omega, \mu)$, then $\varphi_{X}$ is a quasi-concave function (see $[1,11]$ ) and for any $t \in[0, \mu(\Omega))$ we have

$$
\varphi_{X}(t) \varphi_{X^{\prime}}(t)=t .
$$

A non-negative function $q:[0, a) \rightarrow[0, \infty)$ defined on $[0, a)$ with $a \in(0, \infty]$ is called quasi-concave if it is non-decreasing on $[0, a), q(0)=0$ and $t \mapsto q(t) / t$ is nonincreasing on $(0, a)$. Notice also that if $q$ is a quasi-concave function on $[0, a)$, then the function $\widehat{q}:[0, a) \rightarrow[0, \infty)$ defined for $t \in[0, a)$ with

$$
\widehat{q}(t):=\inf \left\{\left(1+\frac{t}{s}\right) q(s): s \in(0, a)\right\}
$$

is a concave function and satisfies conditions $q(t) \leq \widehat{q}(t) \leq 2 q(t)$ for all $t \in[0, a)$. The set of all quasi-concave functions on $[0, \infty)$ will be denoted by $\mathcal{P}$. If in addition $q(t) \rightarrow \infty$ as $t \rightarrow \infty$ then we write $q \in \mathcal{P}_{\infty}$.

The following proposition is a slightly more general version of the result obtained in [17, Proposition 1.5].

Proposition 3.1. Let $X \neq L^{\infty}$ be a maximal symmetric space on $\mathbf{T}, Y$ be a Banach lattice on $\mathbf{T}$ and $\varphi \in \Phi$. Then the following statements are equivalent:

(i) $\widetilde{C}_{\varphi}$ defines an order bounded operator from $H X$ into $Y$.

(ii) $\varphi_{X}(1-|\widetilde{\varphi}|)^{-1} \in Y$. 
Proof. (i) $\Longrightarrow$ (ii). Assume that $\widetilde{C}_{\varphi}: H X \rightarrow Y$ is order bounded. Then there exists a non-negative $g \in Y$ such that the following inequality holds a.e. on $\mathbf{T}$

$$
\left|\widetilde{C_{\varphi} f}\right| \leq g, \quad f \in B_{H X} .
$$

Let $P[g]$ be the Poisson integral of $g$, that is $P[g](z)=\int_{\mathbf{T}} P_{z}(\xi) g(\xi) \mathrm{d} m$. We have

$$
|f(\varphi(z))|=\left|C_{\varphi} f(z)\right| \leq P[g](z), \quad f \in B_{H X}, z \in \mathbf{D} .
$$

Now we use Lemma 1.2 from [17] to obtain the inequalities

$$
\varphi_{X}(1-|\varphi(z)|)^{-1} \leq 4\left\|\delta_{\varphi(z)}\right\|_{(H X)^{*}} \leq 4 P[g](z), \quad z \in \mathbf{D}
$$

where $\delta_{z}$ is a point evaluation functional on $H X$. Notice that if $X \neq L^{\infty}$, then $\lim _{t \rightarrow 0^{+}} \varphi_{X}(t)=0$. If $t \in \mathbf{T}$ is such that $\lim _{r \rightarrow 1^{+}} P[g]\left(r e^{i t}\right)=g\left(e^{i t}\right)<+\infty$, we deduce from the above inequality applied to $z=r e^{i t}$ with $0<r<1$, that

$$
\left|\widetilde{\varphi}\left(e^{i t}\right)\right|<1 \text { and } \varphi_{X}\left(1-\left|\widetilde{\varphi}\left(e^{i t}\right)\right|\right)^{-1} \leq 4 g\left(e^{i t}\right),
$$

by the continuity of $\varphi_{X}$. That is we have $\varphi_{X}(1-|\widetilde{\varphi}|)^{-1} \leq 4 g$ almost everywhere and so $\varphi_{X}(1-|\widetilde{\varphi}|)^{-1} \in X$.

(ii) $\Longrightarrow$ (i). Suppose that $\varphi_{X}(1-|\widetilde{\varphi}|)^{-1} \in Y$. This implies in particular that $\varphi \in \widetilde{\Phi}$. But then for all $f \in B_{H X}$, by the continuity of $f$ in $\mathbf{D}$ we have when $\left|\widetilde{\varphi}\left(e^{i t}\right)\right|<1$

$$
\left|\widetilde{C_{\varphi} f}\left(e^{i t}\right)\right|=\lim _{r \rightarrow 1^{-}}\left|C_{\varphi} f\left(r e^{i t}\right)\right|=\lim _{r \rightarrow 1^{-}}\left|f\left(\varphi\left(r e^{i t}\right)\right)\right|=\left|f\left(\widetilde{\varphi}\left(e^{i t}\right)\right)\right| \leq\left\|\delta_{\widetilde{\varphi}\left(e^{i t}\right)}\right\|_{(H X)^{*}}
$$

Now it follows from [17, Lemma 1.2(ii)] that

$$
\left|\widetilde{C_{\varphi} f}\left(e^{i t}\right)\right| \leq 2 \varphi_{X}\left(1-\left|\widetilde{\varphi}\left(e^{i t}\right)\right|\right)^{-1} .
$$

Thus $\left|\widetilde{C}_{\varphi} f\right|=\left|\widetilde{C_{\varphi} f}\right| \leq 2 \varphi_{X}(1-|\widetilde{\varphi}|)^{-1}$ a.e. on $\mathbf{T}$, for all $f \in B_{H X}$, and so $\widetilde{C}_{\varphi}$ is order bounded. This completes the proof.

Now we will study order bounded composition operators on growth spaces (see Introduction for the definition). In what follows we will use the Matuszewska-Orlicz indices $\alpha_{f}$ and $\beta_{f}$ defined for every $f \in \mathcal{B}$, where $\mathcal{B}$ denotes the class of all measurable functions $f:(0, \infty) \rightarrow(0, \infty)$ such that

$$
\bar{f}(t):=\sup _{s>0} \frac{f(s t)}{f(s)}<\infty, \quad t>0
$$

and $\bar{f}$ is measurable. For $f \in \mathcal{B}$ we put (see [18])

$$
\alpha_{f}:=\sup _{0<t<1} \frac{\log \bar{f}(t)}{\log t}, \quad \beta_{f}:=\inf _{t>1} \frac{\log \bar{f}(t)}{\log t} .
$$

We note that for given $s \in(0, \infty)$ and for $\alpha \in \mathbf{R}$ the function $f:(0, \infty) \rightarrow(0, \infty)$ defined by $f(t)=t^{s}(1+|\log t|)^{\alpha}$ satisfies the following: $\bar{f}(t)=t^{s}(1+|\log t|)^{|\alpha|}$ and $\alpha_{f}=\beta_{f}=s$.

Let $f, g:[0, \infty) \rightarrow(0, \infty)$. We say that $f$ and $g$ are equivalent (and write $f \asymp g$ ), whenever for some $a, b>0, a^{-1} f\left(b^{-1} t\right) \leq g(t) \leq a f(b t)$ for all $t>0$. It is easy to see that $f, g \in \mathcal{B}$ with $f \asymp g$ implies that $\alpha_{f}=\alpha_{g}$ and $\beta_{f}=\beta_{g}$. If $f:[0, \infty) \rightarrow[0, \infty)$ is such that $f(1)=1$, then $f$ is called normalized.

Throughout the rest of the paper, we write $f \in \mathcal{B}_{0}$ whenever $f \in \mathcal{B}$ and $0<$ $\alpha_{f} \leq \beta_{f}<1$. 
We will need the following formula which holds for all $\alpha>0$

$$
\sum_{n=0}^{\infty} 2^{n \alpha} r^{2^{n}}=O\left((1-r)^{-\alpha}\right), \quad 0 \leq r<1
$$

This formula is known (see [4, p. 66]), nonetheless for the sake of completeness we justify it here. The above estimate follows from the following equality

$$
\int_{0}^{\infty} 2^{x \alpha} r^{2^{x}} \mathrm{~d} x=\frac{1}{\log 2} \int_{1}^{\infty} e^{u \log r} u^{\alpha-1} \mathrm{~d} u .
$$

Since for $r \in(0,1), \log r<r-1$, the integral on the right hand side of equality is dominated by

$$
\int_{1}^{\infty} e^{-(1-r) u} u^{\alpha-1} \mathrm{~d} u=(1-r)^{-\alpha} \int_{1-r}^{\infty} e^{-t} t^{\alpha-1} \mathrm{~d} t \leq C_{\alpha}(1-r)^{-\alpha}
$$

and so the required estimate follows.

Before we state and prove the following lemma we recall that the Rademacher functions $r_{n}:[0,1] \rightarrow \mathbf{R}$ are given by $r_{n}(t)=\operatorname{sgn} \sin \left(2^{n} \pi t\right)$ for each integer $n \geq 0$ and every $t \in[0,1]$. For a function $f:[0, \infty) \rightarrow[0, \infty)$ we define the function $f_{*}:[0, \infty) \rightarrow[0, \infty)$ by $f_{*}(0):=0$ and $f_{*}(t):=1 / f(1 / t)$ for all $t>0$.

Lemma 3.2. Let $\psi \in \mathcal{B}$ be a normalized increasing continuous function with $\alpha_{\psi}>0$. For every $t \in[0,1]$ the functions $f_{t}$ defined by

$$
f_{t}(z)=\sum_{n=0}^{\infty} r_{n}(t) \psi\left(2^{n}\right) z^{2^{n}}, \quad z \in \mathbf{D}
$$

belongs to $X_{\phi}$, where $\phi$ is defined by $\phi(r)=\psi_{*}(1-r)$ for all $r \in[0,1]$. Moreover we have $\sup \left\{\left\|f_{t}\right\|_{X_{\phi}}: t \in[0,1]\right\}<\infty$.

Proof. We will need the following known technical result (see, e.g., [16, Proposition 5.1]) which states that if $f \in \mathcal{B}$, then there exist real numbers $\alpha$, $\beta$ with $\alpha<\alpha_{f} \leq \beta_{f}<\beta$ and a concave function $\rho \in \mathcal{B}_{0}$ such that

$$
f(t) \asymp t^{\alpha} \rho\left(t^{\beta-\alpha}\right) .
$$

Since $\alpha_{\psi}>0, \psi(t) \rightarrow \infty$ as $t \rightarrow \infty$. Thus our hypotheses imply that $\phi$ satisfies all required conditions in the definition of $X_{\phi}$. By the mentioned technical result, we may assume without loss of generality that there exist positive constants $\alpha, \beta$ with $\alpha<\beta$ and a concave function $\rho \in \mathcal{P}$, such that

$$
\psi(t)=t^{\alpha} \rho\left(t^{\beta} / t^{\alpha}\right), \quad t>0 .
$$

We now let $\Psi(s, t)=s \rho(t / s)$ for all $s, t>0$. Observe that $\Psi(\lambda s, \lambda t)=\lambda \Psi(s, t)$ for $\lambda, s, t>0$. Thus $\Psi:(0, \infty) \times(0, \infty) \rightarrow(0, \infty)$ is a concave function satisfying

$$
\psi(t)=\Psi\left(t^{\alpha}, t^{\beta}\right), \quad t>0 .
$$

By concavity of $\Psi$, it follows easily that for any finite sets $\left\{s_{0}, \ldots, s_{m}\right\},\left\{t_{0}, \ldots, t_{m}\right\}$ of positive numbers we have

$$
\sum_{k=0}^{m} \Psi\left(s_{k}, t_{k}\right) \leq \Psi\left(\sum_{k=0}^{m} s_{k}, \sum_{k=0}^{m} t_{k}\right) .
$$


Then by the formula $(*)$, for every $\alpha>0$, there exists a constant $C(\alpha)>0$ such that the following estimate holds

$$
\sum_{n=0}^{\infty} 2^{\alpha n}|z|^{2^{n}} \leq \frac{C(\alpha)}{(1-|z|)^{\alpha}}, \quad z \in \mathbf{D}
$$

Put $C=\max \{C(\alpha), C(\beta)\}$ and choose $t \in[0,1]$ and $z \in \mathbf{D}$. Then combining the above estimates we get that

$$
\begin{aligned}
\left|f_{t}(z)\right| & \leq \sum_{n=0}^{\infty} \psi\left(2^{n}\right)|z|^{2^{n}}=\sum_{n=0}^{\infty} \Psi\left(2^{\alpha n}|z|^{2^{n}}, 2^{\beta n}|z|^{2^{n}}\right) \leq \Psi\left(\sum_{n=0}^{\infty} 2^{\alpha n}|z|^{2^{n}}, \sum_{n=0}^{\infty} 2^{\beta n}|z|^{2^{n}}\right) \\
& \leq C \Psi\left((1-|z|)^{-\alpha},(1-|z|)^{-\beta}\right)=C \psi\left((1-|z|)^{-1}\right) .
\end{aligned}
$$

In consequence $f_{t} \in X_{\phi}$ with $\sup \left\{\left\|f_{t}\right\|_{X_{\phi}}: t \in[0,1]\right\} \leq C$.

In the sequel we will use the classical Khintchine inequality (see [15, Theorem 2.b.3]), which states that for every $p \in(0, \infty)$ there exist constants $A_{p}, B_{p}>0$ such that for any choice of scalars $\left\{a_{k}\right\}_{k=1}^{n}$, we have

$$
A_{p}\left(\sum_{k=1}^{n}\left|a_{k}\right|^{2}\right)^{1 / 2} \leq\left(\int_{0}^{1}\left|\sum_{k=1}^{\infty} r_{k}(t) a_{k}\right|^{p} \mathrm{~d} t\right)^{1 / p} \leq B_{p}\left(\sum_{k=1}^{n}\left|a_{k}\right|^{2}\right)^{1 / 2}
$$

We recall also that a quasi-Banach lattice $X$ is said to be $p$-convex, $p \in(0, \infty)$, if there is positive constant $C>0$ such that

$$
\left\|\left(\sum_{k=1}^{n}\left|x_{k}\right|^{p}\right)^{1 / p}\right\|_{X} \leq C\left(\sum_{k=1}^{n}\left\|x_{i}\right\|_{X}^{p}\right)^{1 / p}
$$

for every choice of vectors $x_{1}, \ldots, x_{n} \in X$. The least $C$ satisfying the above inequality is denoted by $M^{(p)}(X)$. A quasi-Banach lattice $X$ is said to have non-trivial convexity if $X$ is $p$-convex for some $0<p<\infty$.

The following lemma will play a key role in the proof of the main theorem of this section.

Lemma 3.3. Let $Y$ be a maximal quasi-Banach lattice on $\mathbf{T}$ with non-trivial convexity and let $\psi \in \mathcal{P}_{\infty}$ be a normalized function. If $\widetilde{C}_{\varphi}$ with $\varphi \in \widetilde{\Phi}$ defines a bounded operator from $X_{\phi}$ into $Y$, where $\phi(r)=\psi_{*}(1-r)$ for all $r \in[0,1]$, then

$$
\left(\sum_{n=0}^{\infty} \psi\left(2^{n}\right)^{2}|\widetilde{\varphi}|^{2^{n+1}}\right)^{1 / 2} \in Y
$$

Proof. Suppose that a quasi-Banach lattice $Y$ is $p$-convex for some $p \in(0, \infty)$. Combining monotonicity of the quasi-norm and its $p$-convexity with the Khintchin inequality (consult the proof of Theorem 1.d.6 in [15]) we get that for any choice of finite set of elements $y_{1}, \ldots, y_{n} \in Y$ the following inequalities hold

$$
\begin{aligned}
A_{p}\left\|\left(\sum_{k=1}^{n}\left|y_{k}\right|^{2}\right)^{1 / 2}\right\|_{Y} & \leq\left\|\left(\int_{0}^{1}\left|\sum_{k=1}^{n} r_{k}(t) y_{k}\right|_{Y}^{p} \mathrm{~d} t\right)^{1 / p}\right\|_{Y} \\
& \leq M^{(p)}(Y)\left(\int_{0}^{1}\left\|\sum_{k=1}^{n} r_{k}(t) y_{k}\right\|_{Y}^{p} \mathrm{~d} t\right)^{1 / p}
\end{aligned}
$$


Since $Y$ is maximal and $f_{t} \in X_{\phi}$ for every $t \in[0,1]$ by Lemma 3.2, we obtain

$$
\begin{aligned}
\left\|\widetilde{C}_{\varphi}\right\| \sup _{t \in[0,1]}\left\|f_{t}\right\|_{X_{\phi}} & \geq\left(\int_{0}^{1}\left\|\widetilde{C}_{\varphi} f_{t}\right\|^{p} \mathrm{~d} t\right)^{1 / p}=\left(\int_{0}^{1}\left\|\sum_{n=0}^{\infty} r_{n}(t) \psi\left(2^{n}\right) \widetilde{\varphi}^{2^{n}}\right\|_{Y}^{p} \mathrm{~d} t\right)^{1 / p} \\
& \geq A_{p} M^{(p)}(Y)^{-1}\left\|\left(\sum_{n=0}^{\infty} \psi\left(2^{n}\right)^{2}|\widetilde{\varphi}|^{2^{n+1}}\right)^{1 / 2}\right\|_{Y} .
\end{aligned}
$$

This completes the proof.

We are ready to prove the main theorem of this section, where we characterize the order bounded composition operator on the growth spaces $X_{\phi}$.

Theorem 3.4. Let $Y$ be a quasi-Banach lattice on $\mathbf{T}$ with non-trivial convexity. Assume that $\psi \in \mathcal{P}_{\infty}$ is normalized function with $\alpha_{\psi}>0$. Let $\varphi \in \widetilde{\Phi}$ and $\phi:[0,1] \rightarrow$ $\mathbf{R}$ be given by $\phi(r)=\psi_{*}(1-r), r \in[0,1]$. The following statements are equivalent:

(i) $\psi\left((1-|\widetilde{\varphi}|)^{-1}\right) \in Y$.

(ii) $\widetilde{C}_{\varphi}$ defines an order bounded operator from $X_{\phi}$ into $Y$.

(iii) $\widetilde{C}_{\varphi}$ defines a bounded operator from $X_{\phi}$ into $Y$.

Proof. For every $f \in B_{X_{\phi}}$ we have

$$
|f(z)| \leq \psi\left((1-|z|)^{-1}\right), \quad z \in \mathbf{D}
$$

and so

$$
\widetilde{C}_{\varphi}(f) \leq \psi\left((1-|\tilde{\varphi}|)^{-1}\right)
$$

This shows that (i) implies (ii). The implication (ii) $\Longrightarrow$ (iii) is trivial.

(iii) $\Longrightarrow$ (i). We claim that for every quasi-concave function $\psi:[0, \infty) \rightarrow[0, \infty)$ the following inequality holds

$$
\left(\sum_{n=0}^{\infty} \psi\left(2^{n}\right)^{2} r^{2^{n+1}}\right)^{1 / 2} \geq \frac{1}{16} \psi\left((1-r)^{-1}\right), \quad r \in[1 / 2,1) .
$$

To see this fix $1 / 2 \leq r<1$ and pick an integer $k \geq 2$ such that $2^{k-1} \leq 1 /(1-r)<2^{k}$. Then we have

$$
r^{2^{k}} \geq\left(1-\frac{2}{2^{k}}\right)^{2^{k}} \geq C=\inf _{x \geq 4}\left(1-\frac{2}{x}\right)^{x} .
$$

Standard calculus shows that the function $(2, \infty) \ni x \mapsto\left(1-\frac{2}{x}\right)^{x}$ is an increasing function and so $C=1 / 16$. Since $\psi$ is non-decreasing,

$$
\left(\sum_{n=0}^{\infty} \psi\left(2^{n}\right)^{2} r^{2^{n+1}}\right)^{1 / 2} \geq \psi\left(2^{k}\right) r^{2^{k}} \geq \frac{1}{16} \psi\left((1-r)^{-1}\right) .
$$

Now observe that the constant function 1 belongs to $X_{\phi}$ and $\widetilde{C}_{\phi} 1=1$. Thus if $\widetilde{C}_{\phi}$ defines a bounded operator from $X_{\phi}$ into $Y$, then 1 belongs to $Y$, and so $L^{\infty} \subset Y$ by the fact that $Y$ is a lattice. To finish the proof we use Lemma 3.3 to conclude that

$$
\psi\left((1-|\widetilde{\varphi}|)^{-1}\right) \chi_{\{|\widetilde{\varphi}|>1 / 2\}} \in Y .
$$

Thus the required statement $\psi\left((1-|\widetilde{\varphi}|)^{-1}\right) \in Y$ follows from $L^{\infty} \subset Y$ and the inequality

$$
\psi\left((1-|\widetilde{\varphi}|)^{-1}\right) \chi_{\{|\widetilde{\varphi}| \leq a\}} \leq \psi\left((1-a)^{-1}\right) \chi_{\mathbf{T}} \in Y, \quad 0<a<1 .
$$




\section{Abstract summing composition operators}

In their remarkable paper [21] Shapiro and Taylor proved that the composition operator $C_{\varphi}$ is a Hilbert-Schmidt operator on $H^{2}$ if and only if $(1-|\widetilde{\varphi}|)^{-1} \in L^{1}(\mathbf{T})$. They also showed that from the mentioned integrability condition it follows that $C_{\varphi}$ is absolutely $p$-summing on $H^{p}$ for all $p \in[1, \infty)$. Note that if $C_{\varphi}: H^{p} \rightarrow H^{p}$, $p \in[2, \infty)$, is absolutely $p$-summing, then $(1-|\widetilde{\varphi}|)^{-1} \in L^{1}(\mathbf{T})$. These results raise a problem of possible characterization of absolutely summing composition operators if $p \in[1,2)$. In this section we provide some results related to this question, namely we study the general summing property in the framework of abstract Hardy spaces. We show also application to the study of absolutely summing and order bounded composition operators on Hardy-Orlicz and Hardy-Lorentz spaces.

Let $(\Omega, \Sigma, \mu)$ be a measure space. Throughout this section $\phi$ will denote an Orlicz function, that is a convex mapping $\phi:[0, \infty) \rightarrow[0, \infty)$ such that $\phi(0)=0$ and $\phi(t)>0$ for all $t>0$. Given an Orlicz function $\phi$, the Orlicz space $L^{\phi}=L^{\phi}(\Omega)$ is the set of all functions $f \in L^{0}(\Omega)$ satisfying

$$
I_{\phi}(\lambda f)=\int_{\Omega} \phi(\lambda|f|) \mathrm{d} \mu<\infty \text { for some } \lambda>0 .
$$

It is well known that $L^{\phi}$ is a Banach lattice equipped with the norm

$$
\|f\|_{\phi}=\inf \left\{\lambda>0: I_{\phi}\left(\frac{f}{\lambda}\right) \leq 1\right\} .
$$

The Orlicz space defined on a purely atomic measure $(\Omega, \mu)$, where $\Omega=\mathbf{Z}_{+}$or $\Omega=\mathbf{N}$ is denoted by $\ell^{\phi}$. For a given Orlicz space $L^{\phi}$ on $\mathbf{T}$ we define the Hardy-Orlicz space $H^{\phi}$ by $H^{\phi}:=H L^{\phi}$.

Let $E$ and $F$ be Banach sequence spaces with $E \hookrightarrow F$ (i.e., $E$ is a linear subspace of $F$ and the embedding is continuous). For every operator $T: X \rightarrow Y$ between Banach spaces and each $n \in \mathbf{N}$ we define

$$
\pi_{F, E}^{n}(T)=\sup \left\{\left\|\sum_{k=1}^{n}\right\| T x_{k}\left\|_{Y} e_{k}\right\|_{F}: \sup _{\left\|x^{*}\right\|_{X^{*}} \leq 1}\left\|\sum_{k=1}^{n}\left\langle x_{k}, x^{*}\right\rangle e_{k}\right\|_{E} \leq 1\right\},
$$

where $e_{k}$ are the standard unit vectors. If $\pi_{F, E}(T):=\sup \left\{\pi_{F, E}^{n}(T): n \in \mathbf{N}\right\}<\infty$, then $T$ is called $(F, E)$-summing. For $F=\ell^{q}$ and $E=\ell^{p}$, with $p, q \in[1, \infty), p \leq q$, we obtain the well-known notion of $(q, p)$-summing operators (resp., $p$-summing in the case when $p=q$ ).

The following result provides sufficient conditions for a composition operator $C_{\varphi}$ to be $\left(\ell_{\phi_{0}}, \ell_{\phi_{1}}\right)$-summing on Hardy-Orlicz spaces. We recall that a function $f:[0, \infty) \rightarrow[0, \infty)$ is called super-mutiplicative if $f(s) f(t) \leq f(s t)$ for all $s, t \geq 0$.

Proposition 4.1. Let $\phi_{0}$ and $\phi_{1}$ be normalized Orlicz functions such that $\phi_{0}$ is super-mutiplicative and there exists a constant $K \geq 1$ such that $\phi_{0}(s t) \leq K \phi_{0}(s) \phi_{1}(t)$ for all $s>0$ and $t \in(0,1]$. If $(1-|\widetilde{\varphi}|)^{-1} \in L^{1}(\mathbf{T})$, then the composition operator $C_{\varphi}: H^{\phi_{0}} \rightarrow H^{\phi_{0}}$ is $\left(\ell_{\phi_{0}}, \ell_{\phi_{1}}\right)$-summing.

Proof. We let $X:=L^{\phi_{0}}(\mathbf{T})$. Then we have $\varphi_{X}(t)=1 / \phi_{0}^{-1}(1 / t), t \in(0,1]$. Our hypothesis implies that there is a subset $A \subset \mathbf{T}$ with $m(A)=0$ such that $\left|\widetilde{\varphi}\left(e^{i t}\right)\right|<1$ for all $t \in A^{c}$, where $A^{c}$ denotes the complement of $A$ in $\mathbf{T}$. It follows from [17, Lemma 1.2(ii)] that

$$
\left|C_{\widetilde{\varphi}} f\left(e^{i t}\right)\right| \leq \frac{2}{\varphi_{X}\left(1-\left|\widetilde{\varphi}\left(e^{i t}\right)\right|\right)}\|f\|_{H X}
$$


for all $t \in A^{c}$ and all $f \in H X$. Let $g$ be given by

$$
g\left(e^{i t}\right)=\frac{2}{\varphi_{X}\left(1-\left|\widetilde{\varphi}\left(e^{i t}\right)\right|\right)}, \quad t \in A^{c} .
$$

The above estimate shows that for every $t \in A^{c}$ the linear functional $x_{t}^{*}$ defined by

$$
x_{t}^{*}(f)=C_{\varphi} f\left(e^{i t}\right) / g\left(e^{i t}\right), \quad f \in H X
$$

is bounded on $H X$ with norm less than or equal to 1 . Now we choose finitely many elements $f_{1}, \ldots, f_{n}$ in $H X$ such that

$$
\sup \left\{\left\|\sum_{k=1}^{n} x^{*}\left(f_{k}\right) e_{k}\right\|_{\ell_{\phi_{1}}}:\left\|x^{*}\right\|_{(H X)^{*}} \leq 1\right\} \leq 1 .
$$

Then for every bounded functional $x^{*}$ on $H X$ with $\left\|x^{*}\right\| \leq 1$, we have

$$
\sum_{k=1}^{n} \phi_{1}\left(\left|x^{*}\left(f_{k}\right)\right|\right) \leq 1
$$

Since $\phi_{0}$ is super-multiplicative, then $\phi_{0}$ satisfies also $\Delta_{2}$-condition (i.e., there exists $c>0$ such that $\phi_{0}(2 t) \leq c \phi_{0}(t)$ for all $\left.t>0\right)$ and so $g \in L^{\phi_{0}}$. Combining with our hypothesis on $\phi_{0}$ and $\phi_{1}$ we have with $\lambda=\|g\|_{X}$,

$$
\sum_{k=1}^{n} \phi_{0}\left(\left|C_{\varphi} f_{k}\left(e^{i t}\right) / K \lambda\right|\right)=\sum_{k=1}^{n} \phi_{0}\left(g\left(e^{i t}\right)\left|x_{t}^{*}\left(f_{k}\right)\right| / K \lambda\right) \leq \phi_{0}\left(g\left(e^{i t}\right) / \lambda\right)
$$

for all $t \in A^{c}$. Integrating both sides of the above inequality over $\mathbf{T}$ yields

$$
\sum_{k=1}^{n} \int_{\mathbf{T}} \phi_{0}\left(\left|C_{\varphi} f_{k}\right| / K \lambda\right) \mathrm{d} m \leq 1
$$

Since $\phi_{0}$ is super-multiplicative, $\phi_{0}\left(\|f\|_{X}\right) \leq \int_{\mathbf{T}} \phi_{0}(|f|) \mathrm{d} m$ for all $f \in X$. This implies that $\sum_{k=1}^{n} \phi_{0}\left(\left\|C_{\varphi} f_{k}\right\|_{X} / K \lambda\right) \leq 1$ and so

$$
\left\|\sum_{k=1}^{n}\right\| C_{\varphi} f_{k}\left\|_{H X} e_{k}\right\|_{\ell_{\phi_{0}}} \leq K\|g\|_{\phi_{0}} .
$$

This completes the proof.

We will study summing properties of composition operators in the general framework of abstract Hardy spaces. To do this we need the notion of Hausdorff-Young pair. Let $X$ be a symmetric space on $\mathbf{T}$ and let $E$ be a Banach sequence lattice on $\mathbf{Z}_{+}$. A pair of spaces $(X, E)$ is said to be a Hausdorff-Young pair on $\mathbf{Z}_{+}$, if there exists a constant $C>0$ such that the following holds: if $f \in X$, then $\{\hat{f}(n)\}_{n \geq 0} \in E$ and $\|\{\hat{f}(n)\}\|_{E} \leq C\|f\|_{X}$. We refer the reader to the article [16] for wider context and general examples of Hausdorff-Young pairs.

Note also that in the following lemma $F(Y)$ denotes the Banach space of sequences $\left\{x_{n}\right\}$ in $Y$ equipped with the norm

$$
\left\|\left\{x_{n}\right\}\right\|_{F(Y)}:=\left\|\left\{\left\|x_{n}\right\|_{Y}\right\}\right\|_{F} .
$$

Lemma 4.2. Let $X, Y$ be Banach lattices on $\mathbf{T}$ and assume that $X$ is an order continuous and maximal symmetric space such that $\left(X^{\prime}, E\right)$ is a Hausdorff-Young pair on $\mathbf{Z}_{+}$. If $\varphi \in \Phi$ and $\widetilde{C}_{\varphi}: H X \rightarrow Y$ is $(F, E)$-summing, then

$$
\left\{|\widetilde{\varphi}|^{n}\right\} \in F(Y) \text {. }
$$


Proof. Let $x^{*}$ be a bounded linear functional on $H X$ with $\left\|x^{*}\right\| \leq 1$. We recall that the linear map $H X \ni f \mapsto \widetilde{f}$ is a linear isometric isomorphism onto a closed subspace of $X$. Thus a natural identification of the dual $X^{*}$ with the Köthe dual combined with the Hahn-Banach theorem yields that there exists $g \in X^{\prime}$ with $\|g\|_{X^{\prime}}=\left\|x^{*}\right\| \leq 1$ such that

$$
x^{*}(f)=\int_{\mathbf{T}} \tilde{f} g \mathrm{~d} m, \quad f \in H X .
$$

This implies that for $\left\{u_{n}\right\}$ given by $u_{n}(z)=z^{n}$ for all $z \in \mathbf{T}$, we get that for some $g \in X^{\prime}$,

$$
x^{*}\left(u_{n}\right)=\widehat{g}(n), \quad n \in \mathbf{Z}_{+} .
$$

From the assumption that $\left(X^{\prime}, E\right)$ is a Hausdorff-Young pair on $\mathbf{Z}_{+}$, it follows that

$$
\left\|\left\{x^{*}\left(u_{n}\right)\right\}\right\|_{E} \leq C\|g\|_{X^{\prime}}
$$

and so $\left\{\left\|\widetilde{C}_{\varphi}\left(u_{n}\right)\right\|_{Y}\right\} \in F$. Since $\left\|\widetilde{C}_{\varphi}\left(u_{n}\right)\right\|_{Y}=\left\||\widetilde{\varphi}|^{n}\right\|_{Y}$ for each $n \in \mathbf{Z}_{+}$, the required statement follows.

Corollary 4.3. Let $X$ be an order continuous and maximal symmetric space on $\mathbf{T}$ such that $\left(X^{\prime}, E\right)$ is a Hausdorff-Young pair on $\mathbf{Z}_{+}$. Assume that a maximal Banach lattice $Y$ on $\mathbf{T}$ is $p$-convex for some $1<p<\infty$. If $\varphi \in \Phi$ and $\widetilde{C}_{\varphi}: H X \rightarrow Y$ is an $\left(\ell^{p}, E\right)$-absolutely summing operator, then

$$
(1-|\widetilde{\varphi}|)^{-1 / p} \in Y \text {. }
$$

Proof. From Lemma 4.2 it follows that $\left\{|\widetilde{\varphi}|^{n}\right\} \in \ell^{p}(Y)$. Thus $p$-convexity of $Y$ implies that there exists a constant $C$ such that

$$
\left\|\left\{\left\||\widetilde{\varphi}|^{n}\right\|_{Y}\right\}\right\|_{\ell^{p}} \geq C\|\|\left\{|\widetilde{\varphi}|^{n}\right\}\left\|_{\ell^{p}}\right\|_{Y} .
$$

In particular this yields $\left|\widetilde{\varphi}\left(e^{i t}\right)\right|<1$ for almost all $t \in \mathbf{T}$, and

$$
\left(1-|\widetilde{\varphi}|^{p}\right)^{-1 / p} \in Y \text {. }
$$

Since $1-r^{p} \leq p(1-r)$ for all $r \in[0,1]$, the required statement follows.

Now we show applications of the obtained results to $p$-summing operators $\widetilde{C}_{\varphi}$.

Corollary 4.4. Let $X$ be an order continuous and maximal symmetric space on $\mathbf{T}$ with fundamental function $\varphi_{X}(t) \asymp t^{1 / p}, p \in[1, \infty)$. Assume that $\left(X^{\prime}, \ell^{p}\right)$ is a Hausdorff-Young pair and $\varphi \in \Phi$. Then the following statements are equivalent:

(i) $\widetilde{C}_{\varphi}$ defines order bounded operator from $H X$ into $L^{p}(\mathbf{T})$.

(ii) $(1-|\widetilde{\varphi}|)^{-1} \in L^{1}(\mathbf{T})$.

(iii) $\widetilde{C}_{\varphi}$ defines $p$-summing operator from $H X$ into $L^{p}(\mathbf{T})$.

Proof. The conditions (i) and (ii) are equivalent by Proposition 3.1. The implication (ii) $\Longrightarrow$ (iii) is a consequence of easily verified fact, that if an operator $T: X \rightarrow L^{p}$ is order bounded, then $T$ is $p$-summing. Finally, the implication (iii) $\Longrightarrow$ (ii) follows from Corollary 4.3.

We finish with applications to Hardy-Lorentz spaces. Given $p \in[1, \infty)$ and $q \in[1, \infty)$ the Lorentz space $L^{p, q}$ on $\mathbf{T}$ consists of those $f \in L^{0}(\mathbf{T})$ for which

$$
\|f\|_{p, q}:=\left(\int_{0}^{1}\left(t^{1 / p} f^{*}(t)\right)^{q} \frac{\mathrm{d} t}{t}\right)^{1 / q}<\infty
$$


(with usual interpretation when $q=\infty$ ). Here $f^{*}$ denotes the decreasing rearrangement of $f$.

It is well known (see, e.g., [1]) that for $1 \leq q \leq p<\infty$, the above formula defines a separable, maximal symmetric space. In the case $1<p<q \leq \infty$ its defines a quasi-norm on $L^{p, q}$ which is known to be equivalent to the norm, say $\|\cdot\|_{p, q}^{*}$ given by $\|f\|_{p, q}^{*}=\left\|f^{* *}\right\|_{p, q}$ for all $f \in L^{p, q}$, where $f^{* *}(t)=\frac{1}{t} \int_{0}^{t} f^{*}(s)$ d $s$ for every $t \in[0,1)$.

We also recall the definition of the sequence Lorentz space $\ell^{p, q}$ (on $\mathbf{Z}_{+}$) given by

$$
\ell^{p, q}=\left\{x=\left\{x_{n}\right\}:\|x\|_{p, q}=\left(\sum_{n=0}^{\infty}\left(n^{1 / p} x_{n}^{*}\right)^{q} \frac{1}{n}\right)^{1 / q}<\infty\right\} .
$$

It is well known the above functionals $\|\cdot\|_{p, q}$ are quasi-norms equivalent to norms under which $L^{p, q}$ and $\ell^{p, q}$ are symmetric spaces (see [1]). If $X=L^{p, q}$ is the Lorentz space defined on $\mathbf{T}$, then the Hardy space $H X$ is denoted by $H_{p, q}$ and is called Hardy-Lorentz space.

Theorem 4.5. Let $2<p<\infty$ and $1 / p+1 / p^{\prime}=1$. For every $\varphi \in \Phi$, the following statements are equivalent:

(i) $\widetilde{C}_{\varphi}$ defines order bounded operator from $H_{p, p^{\prime}}$ into $L^{p}(\mathbf{T})$.

(ii) $(1-|\widetilde{\varphi}|)^{-1} \in L^{1}(\mathbf{T})$.

(iii) $\widetilde{C}_{\varphi}$ defines $p$-summing operator from $H_{p, p^{\prime}}$ into $L^{p}(\mathbf{T})$.

Proof. We will use the "real interpolation theorem" (see [2, Theorem 5.3.1]) from which it follows that if $p_{0}, p_{1} \in[1, \infty]$ and $p_{0} \neq p_{1}$, then

$$
\left(L^{p_{0}}, L^{p_{1}}\right)_{\theta, q}=L^{s, q}, \quad \frac{1}{s}=\frac{1-\theta}{p_{0}}+\frac{\theta}{p_{1}}, \quad \theta \in(0,1)
$$

with equivalent norms. This formula also holds if $L$ is substituted for $\ell$.

Now applying the above interpolation formula to the Fourier transform $\mathcal{F}:\left(L^{1}\right.$, $\left.L^{2}\right) \rightarrow\left(\ell^{\infty}, \ell^{2}\right)$ given by

$$
\mathcal{F}(f):=\{\widehat{f}(n)\}, \quad f \in L^{1}(\mathbf{T})
$$

yields that $\left(L^{s, q}, \ell^{s^{\prime}, q}\right)$ is a Hausdorff-Young pair on $\mathbf{Z}_{+}$for all $s \in(1,2), q \in[1, \infty]$. In particular $\left(L^{p^{\prime}, p}, \ell^{p}\right)$ is a Hausdorff-Young pair.

We use the well-known Köthe duality formulae $\left(L^{r, q}\right)^{\prime}=L^{r^{\prime}, q^{\prime}}$ (which holds up to the equivalence of norms) and hence it follows that $\left(\left(L^{p, p^{\prime}}\right)^{\prime}, \ell^{p}\right)$ is a Hausdorff-Young pair. Note that the fundamental function of $L^{p, p^{\prime}}$ is equivalent to $t^{1 / p}$. To finish the proof it suffices to apply Corollary 4.4.

\section{References}

[1] Bennett, C., and R. Sharpley: Interpolation of operators. - Pure Appl. Math. 129, Academic Press 1988.

[2] Bergh, J., and J. LÖfström: Interpolation spaces. An introduction. - Springer, Berlin 1976.

[3] Domenig, T.: Order bounded and p-summing composition operators. - Contemp. Math. 213, 1998, 27-41.

[4] Duren, P.: Theory of $H^{p}$ spaces. - Academic Press, San Diego 1976.

[5] Hunziker, H., and H. Jarchow: Composition operators which improve integrability. - Math. Nachr. 152, 1991, 83-99.

[6] Hyvärinen, O., M. Lindström, I. Nieminen, and S. ERno: Spectra of weighted composition operators with automorphic symbols. - J. Funct. Anal. 265:8, 2013, 1749-1777. 
[7] Jarchow, H.: Some factorization properties of composition operators. - In: Progress in Functional Analysis (Peñíscola, 1990), North-Holland Math. Stud. 170, North-Holland, Amsterdam, 1992, 405-413.

[8] Jarchow, H.: Absolutely summing composition operators. - In: Functional Analysis (Essen, 1991), Lecture Notes in Pure and Appl. Math. 150, Dekker, New York, 1994, 193-202.

[9] Jarchow, H.: Some functional analytic properties of composition operators. - Quaestiones Math. 18, 1995, 229-256.

[10] JaRchow, H., and R. RiedL: Factorization of composition operators through Bloch type spaces. - Illinois J. Math. 39, 1995, 431-440.

[11] Krein, S. G., Yu. I. Petunin, and E. M. Semenov: Interpolation of linear operators. Transl. Math. Monogr. 54, Amer. Math. Soc., Providence, R.I. 1982.

[12] Lefèvre, P., D. Li, H. Queffélec, and L. Rodríguez-Piazza: Composition operators on Hardy-Orlicz spaces. - Mem. Amer. Math. Soc. 207, Amer. Math. Soc., Providence, R.I., 2010.

[13] Lefèvre, P., D. Li, H. Queffélec, and L. Rodríguez-Piazza: Some new properties of composition operators associated with lens maps. - Israel J. Math. 195:2, 2013, 801-824.

[14] Lefèvre, P., and L. Rodríguez-Piazza: Plongements de Carleson absolument sommants. - C. R. Math. Acad. Sci. Paris 354, 2016, 1209-1213.

[15] Lindenstrauss, J., and L. Tzafriri: Classical Banach spaces II. - Springer, Berlin, 1979.

[16] MastyŁo, M., and P. Mleczko: Solid hulls of quasi-Banach spaces of analytic functions and interpolation. - Nonlinear Anal. 73:1, 2010, 84-98.

[17] Mastylo, M., and L. Rodríguez-Piazza: Carleson measures and embeddings of abstract Hardy spaces into function lattices. - J. Funct. Anal. 268:2, 2015, 902-928.

[18] Matuszewska, W., and W. Orlicz: On certain properties of $\varphi$-functions. - Bull. Acad. Polon. Sci. Ser. Sci. Math. Astronm. Phys. 8, 1960, 439-443.

[19] Rubel, L. A., and A. L. Shields: The second duals of certain spaces of analytic functions. J. Austral. Math. Soc. 11, 1970, 276-280.

[20] Shapiro, J. H.: Composition operators and Schröder's functional equation. - Contemp. Math. 213, 1998, 213-228.

[21] Shapiro, J.H., and P.D. TAYlOR: Compact, nuclear, and Hilbert-Schmidt composition operators on $H^{2}$. - Indiana Univ. Math. J. 23, 1973/74, 471-496.

Received 22 July 2017 • Accepted 10 December 2018 